\title{
A New Method of Edge Crack Suppression in the Rolling Process of Magnesium Alloy Sheet and Study on the Method of Cathodic Protection
}

\author{
Xintao Li $\mathbb{D}^{1},{ }^{1}$ Yanchun Zhu, ${ }^{1}$ Haibin Li, ${ }^{1}$ Yong Niu, ${ }^{1}$ and Chenglong Yang ${ }^{2}$ \\ ${ }^{1}$ College of Electronic Information Engineering, Heavy Machinery Engineering Research Center of Education Ministry, \\ Taiyuan University of Science and Technology, Taiyuan 030024, Shanxi, China \\ ${ }^{2}$ College of Mechatronics Engineering, North University of China, Taiyuan 030051, Shanxi, China
}

Correspondence should be addressed to Xintao Li; 15003519081@163.com

Received 5 December 2019; Revised 9 March 2020; Accepted 23 March 2020; Published 7 May 2020

Academic Editor: Carlos R. Rambo

Copyright (c) 2020 Xintao Li et al. This is an open access article distributed under the Creative Commons Attribution License, which permits unrestricted use, distribution, and reproduction in any medium, provided the original work is properly cited.

\begin{abstract}
A new method is proposed to solve edge cracking and corrosion in rolling process of the magnesium alloy plate. Usually in the process, the edge cracking is caused by the great temperature difference between the edge and the center of the alloy plate and the corrosion is by water in air. The method uses hot air wrapped around the plate to perfectly prevent edge cracking and corrosion. The method also includes cathodic protection for the magnesium alloy based on electrochemistry. The suppression of cathodic protection on the chemical activity of the magnesium alloy was studied. The experiment shows that the new method of edge heating with hot air wrapping is effective to prevent edge cracking and corrosion. Also, the cathodic protection can effectively suppress the chemical activity of the magnesium alloy and improve the surface quality in the rolling process of the magnesium plate, which provides a new concept in rolling process of the magnesium alloy.
\end{abstract}

\section{Introduction}

Magnesium alloys are new materials for the biomedical and automobile industries. The alloys have a lot of properties, such as low density, high specific strength, high specific stiffness and fatigue strength, good thermal conductivity, good damping and vibration reduction, good cutting performance, and easy recycling, that leads to numerous industrial applications [1-3]. The environment control of rolling equipment has a great effect on the quality in the rolling process of the magnesium alloy [4-6].

Firstly, the temperature has a great influence on the forming and cracking quality after rolling [7-10]. There is a large temperature difference between the center and the edge of the magnesium alloy in the rolling process of the magnesium alloy. The temperature results in a metal flow difference between the center and the edge of the alloy. Edge crack occurs when the additional tensile stress on the edge exceeds the fracture strength limit of the magnesium alloy $[11,12]$.
The existing methods for temperature control in the rolling process of the magnesium alloy are induction heating, resistance heating, flame heating, etc. [13]. In the roll heating method, the heating efficiency of the magnesium alloy plate is low. The temperature difference between the center and the edge is difficult to be eliminated, and the problem of friction heating between the associated roll and the magnesium alloy plate as well as the bearing lubrication deterioration of the roll system may also occur. By the resistance heating method, the temperature difference between the center and the edge of local heating cannot be targeted. Although the flame heating method can effectively eliminate the local heating caused by the temperature difference between the center and the edge, the magnesium element in the magnesium alloy has active chemical properties, which is easy to induce the modification of the magnesium alloy and even induce the spontaneous combustion accident of the magnesium alloy.

The heating transfer has three forms: conduction, radiation, and convection based on thermodynamics. After 
performing research and experiment, it was found that the main reason of temperature difference between the center and the edge is the unbalanced dissipation of the plate caused by hot air convection at the edge. Also, another reason is the friction heating between the plate and rollers. Accordingly, an active temperature compensation method using air heating at the edge is proposed in this paper. This method eliminates the temperature difference in rolling process using the temperature compensation effect that the raised hot air wraps on both sides of the plate. So finally, the method prevents edge cracking.

Secondly, the magnesium element in the magnesium alloy has active chemical properties, which can easily react with the chemical elements in the rolling environment [14]. So far, the effect of humidity on the forming of the magnesium alloy in the rolling environment has often been ignored. Above $120^{\circ} \mathrm{C}$, water vapor will accelerate the oxidation speed of magnesium alloys $[15,16]$. According to the chemical properties of magnesium, there are the following reactions:

$$
\begin{aligned}
\mathrm{Mg}+\mathrm{H}_{2} \mathrm{O}+\mathrm{CO}_{2} & =\mathrm{MgCO}_{3}+\mathrm{H}_{2} \\
\mathrm{Mg}+\mathrm{H}_{2} \mathrm{O} & =\mathrm{MgO}+\mathrm{H}_{2} \\
\mathrm{Mg}+2 \mathrm{H}_{2} \mathrm{O} & =\mathrm{Mg}(\mathrm{OH})_{2}+\mathrm{H}_{2}
\end{aligned}
$$

Obviously, magnesium alloys can easily produce hydrogen in high-humidity rolling environment [17-19], as shown in (1)-(3). The ignition temperature of pure hydrogen is $400^{\circ} \mathrm{C}$, and the lowest ignition point of hydrogen in air is $574^{\circ} \mathrm{C}$. It is lower than the ignition points $632-635^{\circ} \mathrm{C}$ of magnesium in air [20]. At the same time, metallic magnesium can be used as a high-energy storage material for hydrogen storage [21]. At about $250^{\circ} \mathrm{C}$ and at normal pressure, magnesium and hydrogen can be produced, which can release hydrogen at low pressure or slightly higher temperature [22]. In the field of electrochemistry, hydrogen can also cause hydrogen corrosion in the form of hydrogen bulging [23-25], hydrogen embrittlement [26-28], and hydrogen corrosion [29, 30]. Hydrogen can be immersed between the lattice of the metal and expand or deform the lattice and finally make the metal brittle that is called as hydrogen embrittlement. In the process of rolling, the embrittlement would result in cracks in the middle of the magnesium alloy that affects the quality of the magnesium alloys [31-33]. Therefore, it is necessary to dehumidify in the rolling environment [34, 35].

Magnesium metal reacts with other substances in the air as follows [36]:

$$
\begin{aligned}
2 \mathrm{Mg}+\mathrm{O}_{2} & =2 \mathrm{MgO} \\
3 \mathrm{Mg}+\mathrm{N}_{2} & =\mathrm{Mg}_{3} \mathrm{~N}_{2} \\
2 \mathrm{Mg}+\mathrm{CO}_{2} & =2 \mathrm{MgO}+\mathrm{C} \\
\mathrm{Mg}+\mathrm{Br}_{2} & =2 \mathrm{MgBr}_{2} \\
2 \mathrm{Mg}+\mathrm{SiO}_{2} & =2 \mathrm{MgO}+\mathrm{Si} \\
\mathrm{Mg}+\mathrm{H}_{2} \mathrm{~S} & =\mathrm{MgS}+\mathrm{H}_{2}
\end{aligned}
$$

Because of so many chemical reactions, active prevention should be used in engineering $[37,38]$. The protection gas sulfur hexafluoride is often used to isolate during the casting of the magnesium alloy [39, 40]. However, this method is used in rolling equipment, so a series of problems may arise. First of all, the cost of rolling process is rising. The protective gas only needs to cover the pouring ladle in casting, but it is obviously difficult to realize on the rolling process with the length in kilometers. The sharp increase in cost naturally affects the scope of application of the magnesium alloy, but it has certain feasibility for small batch or coil rolling. Secondly, the specific heat capacity of the protective gas is lower, so it needs rapid circulation to achieve the edge-wrapped insulation. Thirdly, sulfur hexafluoride gas can cause depletion of the ozone layer [41]. Once a large amount of the protective gas leaks, it will cause environmental hazards. Therefore, more safe and inexpensive protection methods are needed in the magnesium alloy rolling equipment. The cathodic protection method should become an option for the rolling equipment of the magnesium alloy $[42,43]$.

Based on this, the new concept of thermal insulation rolling table equipment and edge crack suppression for the magnesium alloy is put forward in this paper. Combined with the study of cathodic protection, the method realizes to suppress edge crack and hydrogen corrosion [44, 45] in rolling process of the magnesium alloy plate and effectively improve the forming quality of the magnesium plate [46].

\section{Design of Thermal Insulation Device and Working Principle of Cathodic Protection Equipment}

2.1. A Hot Air Edge Temperature Compensation Roller Incubator for Rolling the Magnesium Alloy. According to the basic principle of air flow, the traditional conveying roller table is shown in Figure 1(a). The rapid air flow will be formed on both sides of the magnesium alloy plate. Edge temperature is lower than the center temperature. That is, $T_{\mathrm{e}}<T_{\mathrm{c}}$ ( $T_{\mathrm{c}}$ is the center temperature of the magnesium alloy plate and $T_{\mathrm{e}}$ is the edge temperature of the magnesium alloy plate). According to the basic principle of air flow, the air flow diagram of a heat preservation conveying roller table can be designed, as shown in Figure 1(b).

The basic principle of air flow is as follows:

$$
L_{R}=\frac{p_{1}-p_{2}}{\rho_{m}}+\left(\frac{v_{1}^{2}}{2}-\frac{v_{2}^{2}}{2}\right)+g\left(Z_{1}-Z_{2}\right)+L_{t},
$$

where $L_{R}$ represents the energy consumed by air to overcome flow resistance, $(\mathrm{J} / \mathrm{kg}) ; L_{t}$ is the wind pressure generated by external wind energy, $(\mathrm{J} / \mathrm{kg}) ; p_{1}$ and $p_{2}$ are the parameters of the section which are the absolute static pressure of the air flow, $\left(P_{\mathrm{a}}\right) ; v_{1}$ and $v_{2}$ are the average velocity of cross-section air flow, $(\mathrm{m} / \mathrm{s}) ; \rho_{m}$ is the density of the wind flow, $\left(\mathrm{kg} / \mathrm{m}^{3}\right) ; Z_{1}$ and $Z_{2}$ are the air gravity potential energy parameters, height from datum $(\mathrm{m})$.

According to the above basic principle formula (5), the air flow is shown in Figure 1(b). The edge temperature is 


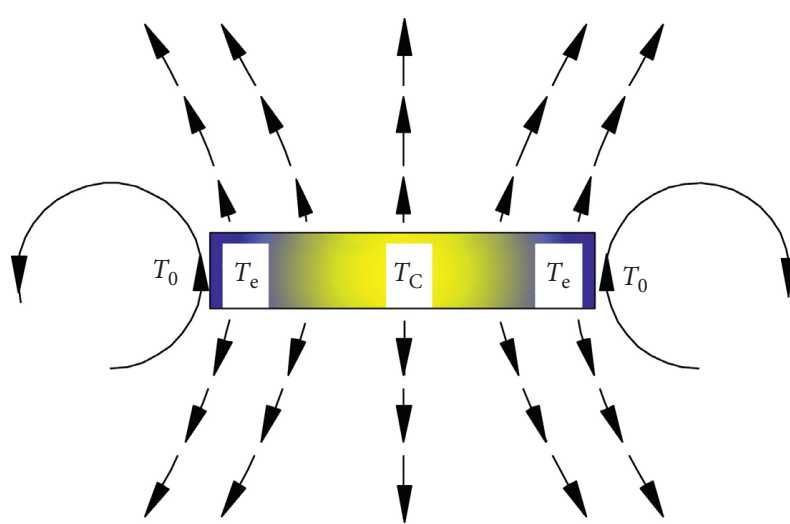

(a)

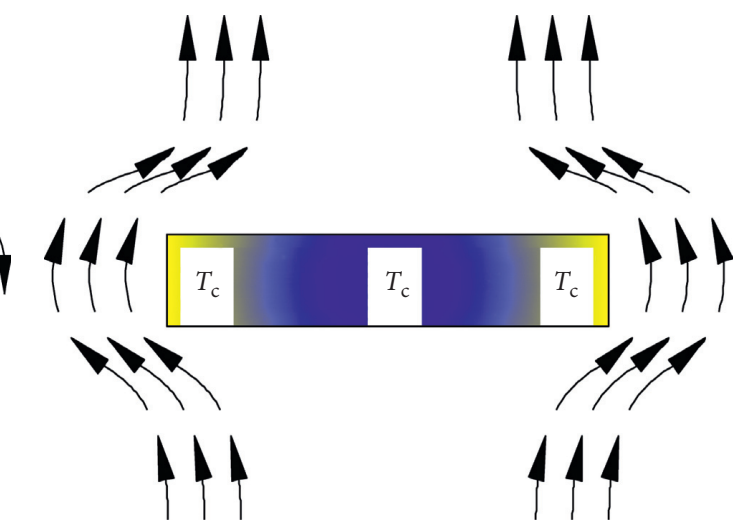

(b)

Figure 1: Air flow diagram of the magnesium alloy plate roller table. (a) Air flow diagram of the traditional conveying roller table. (b) Air flow diagram of the insulation conveying roller table.

equal to the center temperature, $T_{\mathrm{e}}=T_{\mathrm{c}}$. The internal structure of the alloy insulation roller table is shown in Figures 2 and 3.

The architecture of the thermal insulation rolling table equipment for rolling the magnesium alloy is shown in Figures 4 and 5. With the recycling hot air as the heat source $L_{t}(5)$, the new air is heated by the preparation box. The heated hot air is transferred to the lower side of the roller table through filters and pipes. The filter can filter out excess water vapor and metal dust in the air. Then, the hot air is evenly diffused by the air uniform diffuser and flows from both sides of the roller to the upper side (Figures 2 and 3 ). The hot air recycling device is mounted on the top of the conveyor roller table and the frame that with the heat preparation box forms a loop by the pipeline.

In order to achieve the effect of constant temperature of the magnesium alloy plate, temperature feedback control is adopted and pressure and temperature sensors are used in the feedback path.

The theoretical basis of the temperature compensation roller equipment for the magnesium alloy is hot air convection. Because of the obstacle of the magnesium alloy, the hot air produced by the heat preparation box will be wrapped on both sides, thus forming the temperature compensation for the both edges of the magnesium alloy plate. A specific thermal insulation design for wrapping both edges has effectively reduced the rapid temperature loss at the edges of the magnesium alloy.

The working principle of the temperature compensation roller equipment for the magnesium alloy is shown as follows: after filtering and drying, the air is heat up in the heat preparation box. Then, the hot air is evenly diffused from the bottom of the equipment through the pipeline. The hot air then rises and transfers the heat to the magnesium alloy and then returns to the heat preparation box through the collection device and the recycling pipeline. Efficient recycling has achieved the goal of energy saving, emission reduction, and environmental protection.

The temperature compensation roller equipment achieves the purpose of heating at both edges of the magnesium alloy, collects harmful gases and dust, and eliminates potential hazards by the approximate steady temperature field formed by recycling hot air convection. In addition, the control of the valve in the equipment can effectively prevent the damage of the downstream production equipment if the heat preparation box fails. The uniform air diffusion equipment inside effectively prevents chemical reactions such as with oxygen and nitrogen, by directly blowing on the surface of the magnesium alloy, that results in poor surface quality and potential hazards.

Although the design effectively compensates the rapid heat dissipation at the edge by dehumidification and edge insulation, the reaction between magnesium and other air substances cannot be reduced actively.

2.2. Design and Working Principle of Magnesium Alloy Cathodic Protection Device. Xintao Li found that the cathode of the battery is not rusty, so he proposed a new concept of cathodic protection.

The concept of a cathodic protection with an external power supply is shown in Figure 6. Because of the skin effect, a "free electron wall" is formed on the metal surface when an external DC power supply is given (the "free electron wall" is the outer region of the metal surface, which contains a high concentration of free electrons). Due to the existence of the "free electron wall," a microelectric field is formed between the metal surface and the environmental particles. It effectively reduces the possibility that the environmental particles capture the free electrons of the metal materials. Also, the wall realizes the protection of the number of free electrons of the metal materials and thus maintains the chemical properties of the metal. This method is called cathodic protection with an external power supply.

In the conventional cathodic protection method, magnesium is used as the sacrificial anode to protect other metals with low chemical activity [47-50]. In the magnesium alloy rolling process, cathodic protection is needed for the magnesium alloy rolling equipment to reduce its chemical reaction with external materials. The chemical expression is shown as follows: 


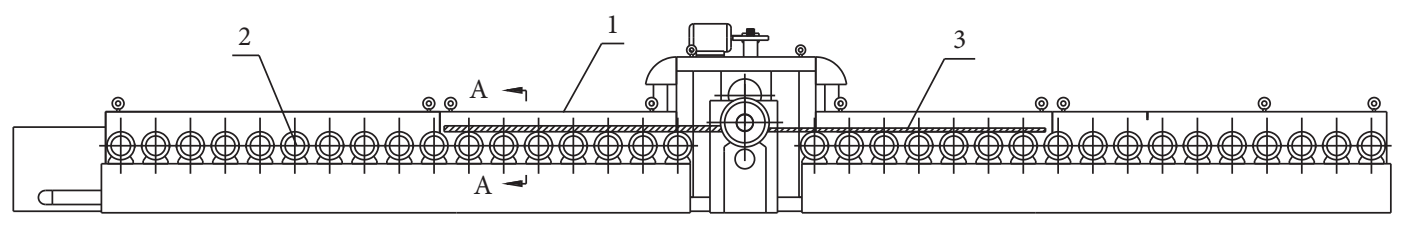

Figure 2: Positive view of the hot air edge temperature compensation roller incubator for rolling the magnesium alloy. 1, roller incubator; 2, roller motor; 3 , magnesium plate.

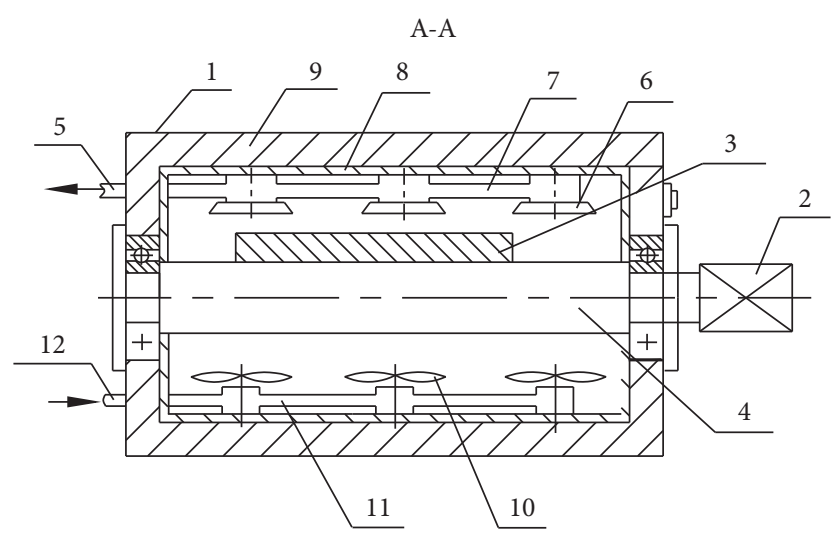

FIgURE 3: Section view of A-A in the structure of the hot air edge temperature compensation roller incubator for rolling the magnesium alloy. 1 , roller incubator; 2 , roller motor; 3 , magnesium plate; 4 , transmission roller; 5 , heat recycling pipe; 6 , heat recovery unit; 7 , heat return pipe; 8 , insulation layer; 9 , insulation box shell; 10, air uniform diffuser; 11, heat transfer branch pipe; 12, hot air pipeline.

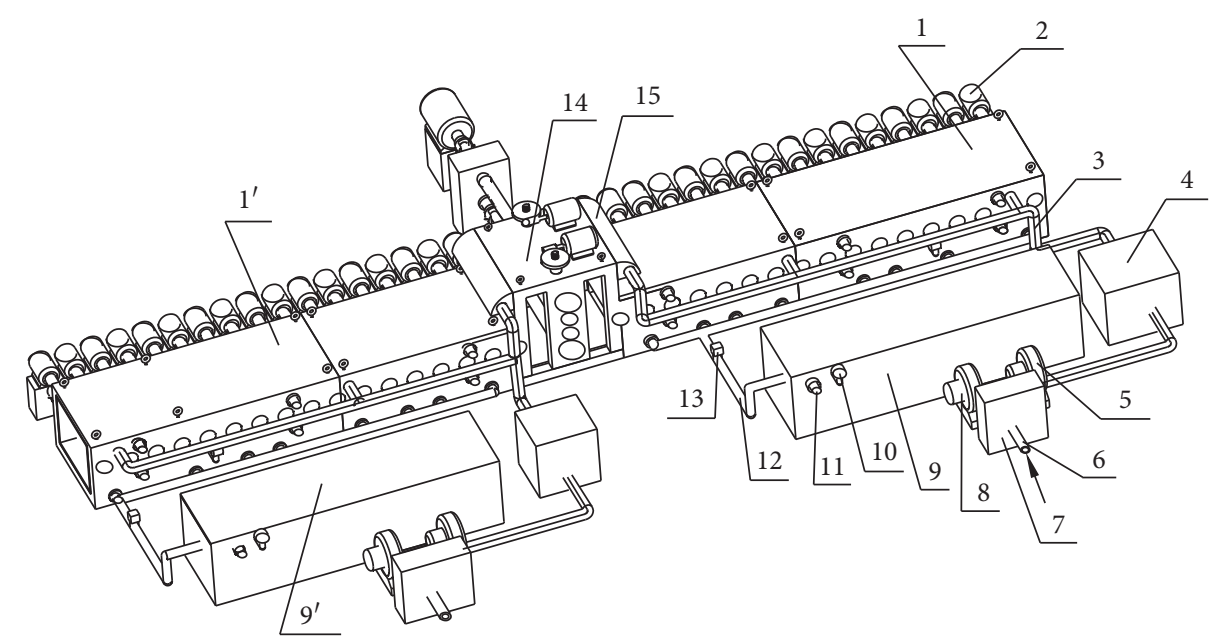

FIGURE 4: A schematic diagram of the three-dimensional structure of the hot air edge temperature compensation roller incubator for rolling the magnesium alloy. 1 , roller table; 2 , roller motor; 3 , heat recycling pipe; 4, filter; 5 and 8 , blower; 6 , replenishment pipeline; 7, filter; 9 , heat preparation box; 10, pressure sensor; 11, temperature sensor; 12 , hot air pipeline; 13, high-temperature speed control valve; 14 , rolling machine; 15 , heat recycling device mounted on the top.

$$
\text { Cathodic protection: } \mathrm{Mg}+\mathrm{e}=\mathrm{Mg}^{-}
$$

The theoretical basis of electrochemical protection is the method of controlling corrosion by utilizing polarization characteristics of the metal electrochemical corrosion process [51-53]. The traditional online cathodic protection principle says that when the magnesium alloy reaches the equilibrium potential, the cathode current is applied again, and the electrode potential of the metal is shifted from the original equilibrium potential to the negative, so that the metal enters the corrosion-free area for protection $[54,55]$.

In the rolling process of the magnesium alloy plate, the magnesium alloy plate as a cathode electrode forms a circuit with the air and the earth. The current is extremely small due to the large resistance of the air. Also, it can be considered as an open circuit. Therefore, smaller cathode power supply can be used in this process. This method is different from the strong current protection in the traditional online cathodic 


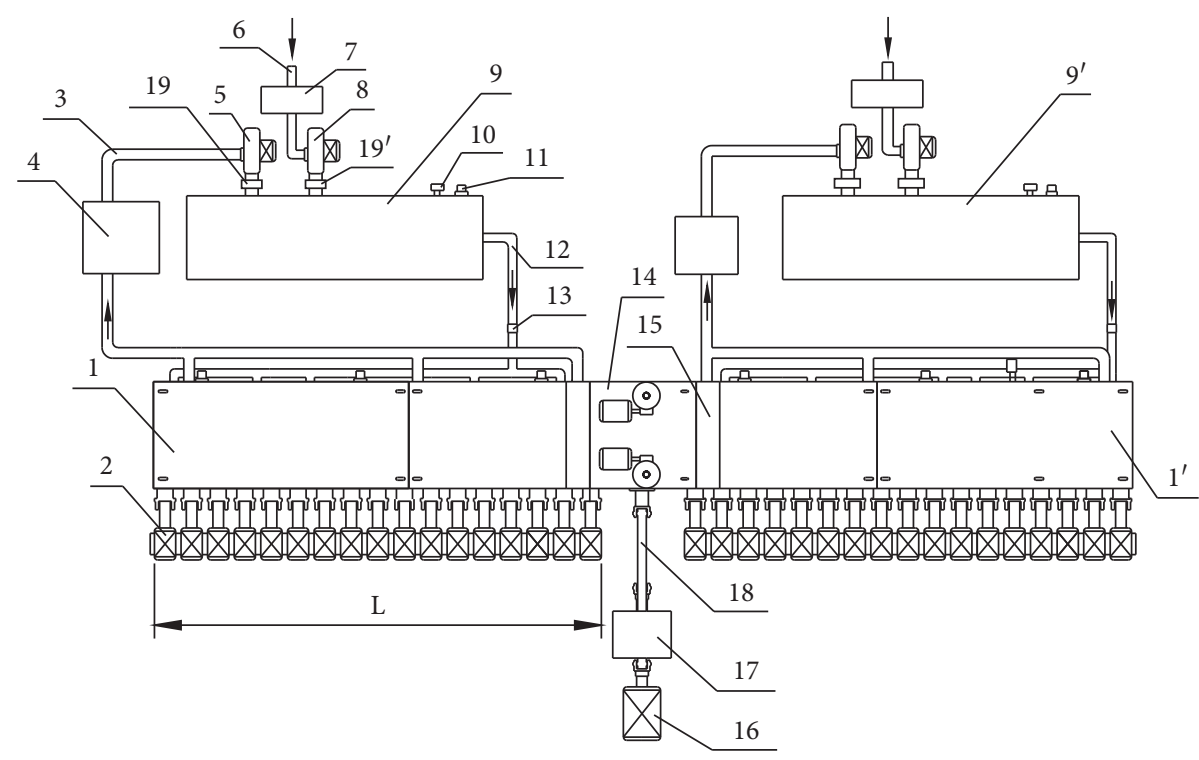

Figure 5: Top view of the hot air edge temperature compensation roller incubator for rolling magnesium alloy. 1, roller table; 2 , roller motor; 3 , heat recovery pipe; 4 , filter; 5 and 8 , blower; 6 , replenishment pipeline; 7, filter; 9 , heat preparation box; 10, pressure sensor; 11 , temperature sensor; 12 , hot gas pipeline; 13 , high-temperature speed control valve; 14 , rolling machine; 15 , heat recycle cover mounted on rolling mill; 16 , rolling mill main motor; 17 , speed reduction box; 18 , kaleidoscope; 19, unidirectional valve.

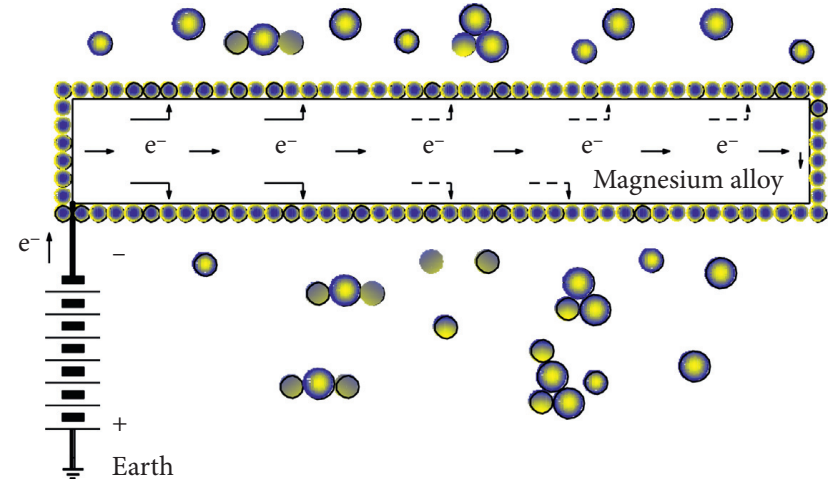

FIGURE 6: Schematic diagram of a cathodic protection method of an external power supply.

protection. The magnesium alloy plate is full of static electricity. According to the skin effect in electromagnetics, negative charges will be accumulated in the surface of the magnesium alloy plate, which is known as negative charge encapsulated cathodic protection or negative charge protection. However, there is a potential difference between the substance in the air and the magnesium alloy plate, which suppress the chemical reaction.

In order to realize the active cathodic protection methods, two methods for negative charge cathodic protection of magnesium alloys are proposed in this paper.

2.2.1. Transmission Roller with Cathodic Protection of Hanging Bare Wire. The hanging bare electric cables are installed on alumina ceramic conveyor rollers on the front and back of the rolling table that is shown in Figures 7 and 8. The upper end of the bare electric cables is connected with the negative electrode of the adjustable power supply, and the lower end is in contact with the magnesium plate. So, the negative electrode of the adjustable power supply finally is connected to the magnesium plate, and the positive electrode is grounded. The electric potential of power supply should be adjusted to the corrosion protection potential of the magnesium alloy, which meets the requirements of the cathodic protection.

The hanging bare electric cable is in contact with the magnesium alloy plate under the influence of gravity in the process. The negative voltage on the magnesium alloy plate will produce the cathode protection. Thus, the protection would reduce the chemical activity of magnesium and improve the quality of magnesium alloys.

\subsubsection{Cathodic Protection Conveyance Roller with Conduc-} tive Roller. Several conductive rollers are installed among alumina ceramic conveyor rollers in the front and back of the magnesium alloy rolling table, as shown in Figure 9. The conductive layer of the conductive roller, shown in Figure 10 , is connected with the negative pole of the adjustable power supply through the sliding ring. The positive pole of the adjustable power supply is grounded. The potential of the adjustable power supply is adjusted to the corrosion potential of the magnesium alloy, which must meets the conditions of the cathodic protection criterion. The magnesium plate is frictionally connected with the conductive layer of several conductive rollers under gravity, so that the voltage of the magnesium plate is negative.

In the transmission, the magnesium alloy plate is frictionally connected with the conductive layer of the conductive roller under gravity. The negative voltage of the plate realizes cathodic protection and reduces the complex 


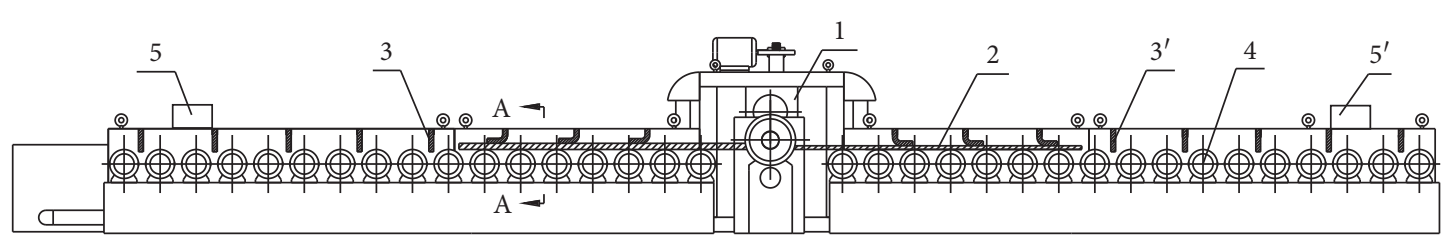

FIGURE 7: Structure diagram of the conveying roller with cathodic protection of a hanging bare wire. 1, rolling mill; 2, magnesium plate; 3 , hanging bare wire; 4 , motor; 5 , adjustable power box.

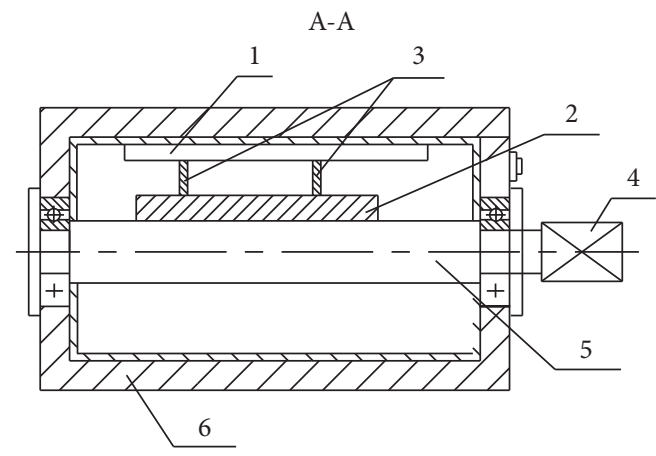

FIGURE 8: Sectional view of conveyor roller A-A with cathodic protection of drooping bare wires. 1, cable bridge; 2, magnesium plate; 3 , hanging bare wire; 4 , motor; 5 , alumina ceramic conveyor roller; 6 , insulation box.

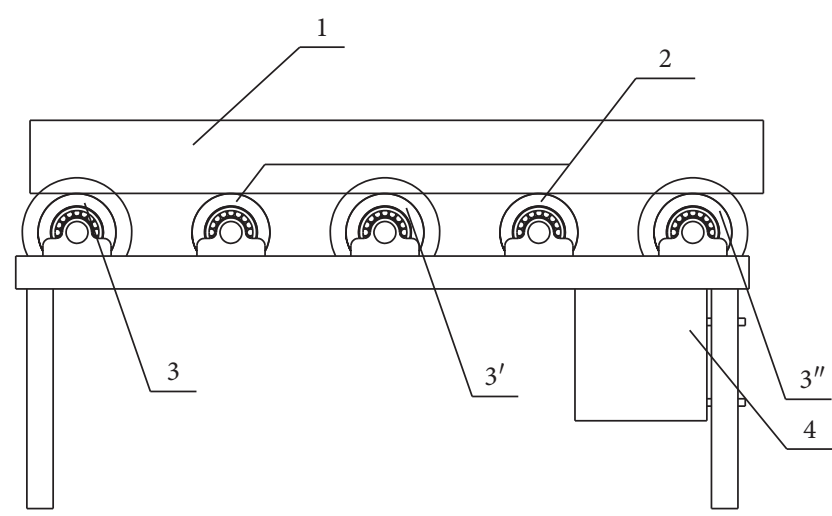

FIGURE 9: Structure diagram of the cathodic protection conveyance roller with a conductive roller. 1, magnesium plate; 2 , alumina ceramic conveyor roller set; $3,3, \ldots, 3 n$, first, second, ..., the nth conductive roller; 4 , adjustable power box.

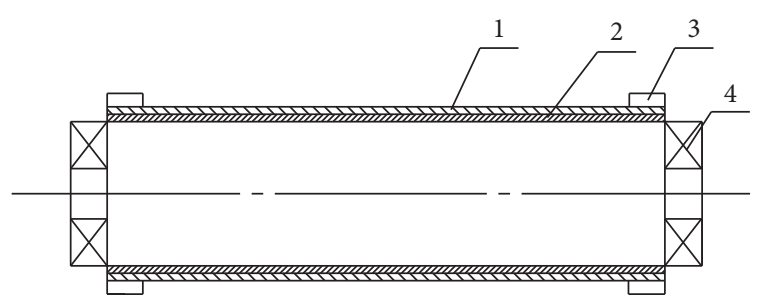

FIgURE 10: Structure diagram of a conductive roller. 1, conductive layer; 2 , insulation layer; 3 , sliding ring; 4 , bearing. chemical reaction between magnesium and external substances.

\section{Design of the Experimental Equipment and Methods}

According to the working principle of the temperature compensation roller equipment, the air-drying box of the general equipment is used to simulate its work. The type of purchased hot air insulation box is a 10 hs electrothermal constant temperature drying box. Because there is no hot air circulating the heating equipment in the laboratory, side heating is used for the heating of the hot air-drying box. In order to observe the heating process of the magnesium alloy effectively, magnesium alloy plates were placed vertically. The temperature difference between the upper and lower parts of the magnesium alloy plate can be clearly observed by the infrared thermometer.

Cathodic protection power supply: an SS3323 trackable DC regulated power supply is selected. Since the cathode protection voltage is applied only to realize the negative electrostatic charge, electrochemical suppression of the formation of chemical reaction conditions between external substances and magnesium alloys can be achieved.

The theoretical standard electrode voltage potential of magnesium is $-2.37 \mathrm{~V}$ (EH) [56]. The selection of cathode voltage for cathodic protection is based on the electrode area of the magnesium alloy plate in the process. Also, the surface of the plate is rarely absolutely smooth during the rolling process. So, the real surface area is 2-3 times its geometric area. Considering the multiple cathode reactions, thermoelectric battery effect, variation of the rolling temperature, resistivity of the heated magnesium alloy, and redundancy of the protective voltage, $-10 \mathrm{~V}$ is selected as the setting value of the cathode voltage.

The nominal composition of the magnesium alloy: the $\mathrm{Mg}-\mathrm{Al}-\mathrm{Zn}$ alloy $\mathrm{Mg}_{2} \mathrm{Al}_{1} \mathrm{Zn}$ plate is selected. The aluminium in the plate is used to suppress the hydrogen evolution and self-corrosion as the magnesium anode, and the zinc is used to reduce the voltage "lag" effect caused by aluminium.

Dimensions are as follows: $200 * 200 * 5 \mathrm{~mm}$ and $200 * 200 * 3 \mathrm{~mm}$.

Temperature measuring equipment which was used is the thermal imager FlukeE4.

Experimental method: magnesium alloy plate specimens were placed vertically in a $10 \mathrm{hs}$ electrothermal constant 
temperature drying box, as shown in Figure 10. No cathode protection voltage was applied, and power was offed to cool down after reaching the highest temperature. During this process, the Fluke thermal imager was used to measure the temperature and record the temperature field distribution. Then, another sample of the magnesium alloy plate was placed vertically in a 10 hs electrothermal constant temperature drying box and cathodic protection voltage was applied, as shown in Figure 11. The steps were repeated as given above, and the temperature field distribution was recorded.

The experimental results of two magnesium alloy plate specimens were randomly sampled by a third party. Under the scanning electron microscope (SEM) and EDS analysis, the effect of cathodic protection voltage on the magnesium alloy was observed.

The experimental steps are as follows:

(1) The magnesium alloy plate was erected in the airdrying box, and the electrothermal heating drying box was set at 400 degree Celsius. The temperature was measured by the Fluke thermometer E4, including the process of heating and cooling, and the temperature distribution of the magnesium alloy plate was observed.

(2) Cathodic protection power test, SS3323, was used to track the DC power supply, the positive electrode was grounded, and the negative electrode was connected to the magnesium alloy metal plate through a conductive clamp. The voltage was set at $10 \mathrm{~V}$, and the heating experiment was conducted. The Fluke thermometer E4 was used to measure the temperature field distribution of the magnesium alloy plate.

(3) After the heating experiment was completed, the surface difference of the metal was visually measured. The samples were randomly sampled by a third party and then analyzed by scanning electron microscopy (SEM) and EDS to compare the differences between the samples.

(4) The experimental data were analyzed and compared, and the experimental conclusion was drawn.

\section{Experimental Results and Analysis}

4.1. Analysis of Air Flow on Infrared Thermal Imaging Test of Magnesium Alloy Plate. As shown in Figure 11, the thermal conductivity of magnesium alloys is good, and it is greatly affected by the temperature distribution of heated air. The experimental results show that the temperature gradient of the magnesium alloy plate decreases from the top to the bottom, and the temperature difference is highly likely to occur.

It can be seen from Figure 12 that during the cooling process of hot air, the gradient of the upper and lower temperature of the hot air can still be seen. At the same time, the surface colour changes significantly.

In order to better show the hot air heating process of the magnesium alloy, a thinner magnesium alloy plate of $3 \mathrm{~mm}$ was selected and the above experiments were conducted by cathodic protection.

As shown in Figure 13, it can be clearly seen that the $3 \mathrm{~mm}$ magnesium alloy plate is more obviously affected by the heating of heated air and more intuitively illustrates the uniformity of hot air, which has an effect on the temperature of the magnesium alloy plate.

4.2. Improvement of Corrosion by Cathodic Protection. The superficial morphology of magnesium alloys is observed by scanning electron microscopy (SEM), as shown in Figures 14-17. The magnesium alloy plates with the $\mathrm{Mg}_{2} \mathrm{Al}_{1} \mathrm{Zn}$ model were placed in a hot air dryer and heated, one piece without cathode protection negative voltage (Figures 14 and 15) and the other with cathode protection negative voltage (Figures 16 and 17). The experimental results show that the superficial morphology of the magnesium alloy with cathodic protection voltage is superior to that of the magnesium alloy without cathodic protection voltage. It can be proved that it is feasible to control the hightemperature chemical reaction of the magnesium alloy by online cathodic protection.

That is to say, cathodic protection can effectively control the chemical activity of magnesium alloy metals. Its theoretical basis is to increase the number of negative electrons of the active metal-magnesium alloy through external power supply. Under the skin effect, electrons of the magnesium alloy plate gather on the metal surface, form potential difference with the outside air, and suppress the reaction of the magnesium metal.

In the field of chemistry, the transient process of chemical reaction is deeply analyzed. The microscopic reactions of the same substance are inconsistent under the same experimental conditions (Figures 14 and 15). In this experiment, the edge temperature of hot air is higher than the center temperature, and the temperature of the yellow flame is higher than that of the red flame (Figure 13). In addition, there is a deep scratch in the upper right corner (Figure 14) and a deep scratch in Figure 15. That is to say, the process of heating chemical reaction, is a process of microchange. As time goes on, the magnesium oxide powder is gradually formed and the metal surface is released. Therefore, the heating efficiency also has an effect on the superficial morphology of the rolled magnesium alloy.

As shown in Figures 14-16, the effect of cathodic protection on the magnesium alloy plate is very obvious. Under the microscopic structure, it can be seen that applying cathodic protection voltage (Figure 16) can effectively suppress the chemical reaction between the magnesium alloy and the outside world. Not only irregular morphology of obvious chemical reaction (Figure 14), but also impurity precipitation (Figure 15) appeared on the magnesium alloy plates without cathodic protection. During the rolling process of the magnesium alloy plate, the hard particles are pressed into the magnesium alloy plate by the rolling force of the roller, and the cracks will occur randomly, resulting in the decline of product quality.

In order to further understand the composition, EDS analysis was carried out. It can be seen that the loose 


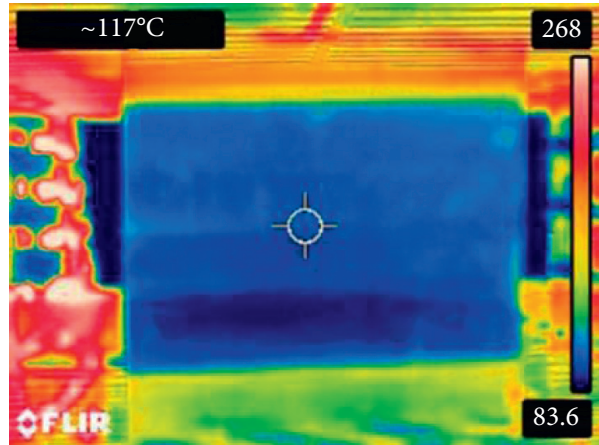

(a)

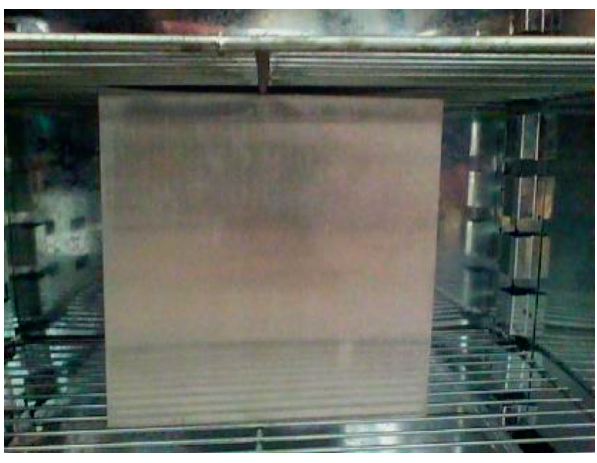

(b)

FIGURE 11: Hot air heating experiment of the electro-magnesium alloy without cathodic protection.

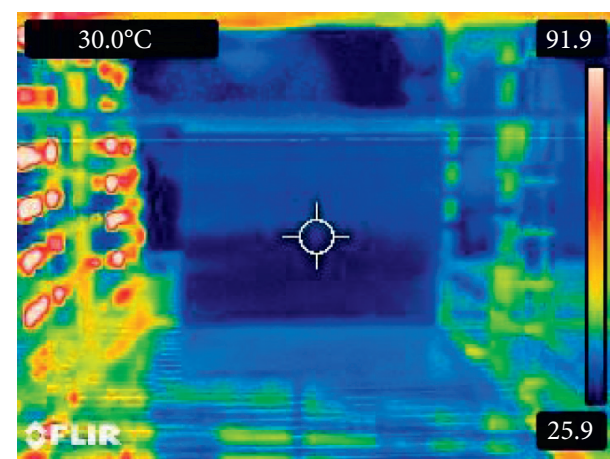

(a)

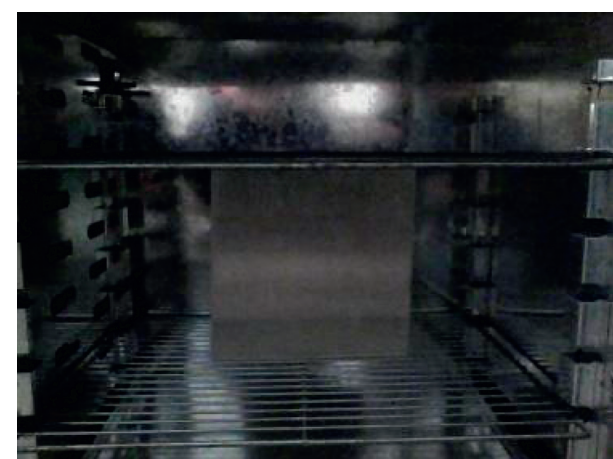

(b)

FIgURE 12: Experimental cooling of hot air heating for the electro-magnesium alloy without cathodic protection.

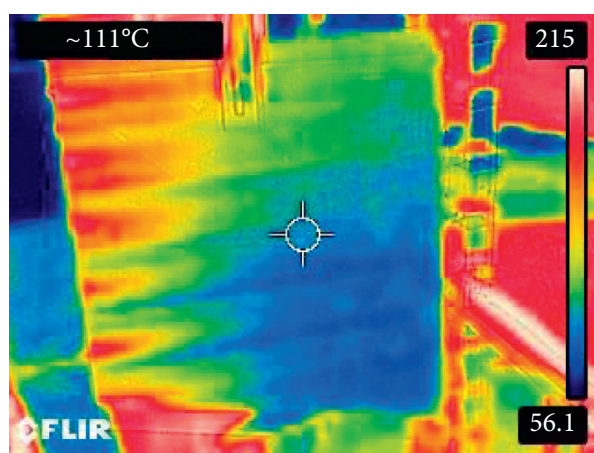

(a)

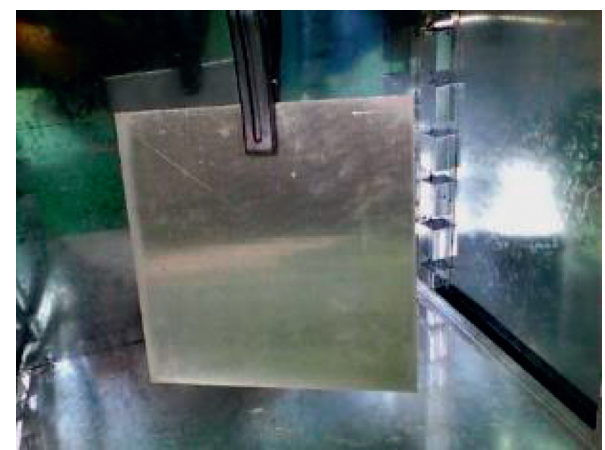

(b)

FIGURE 13: Hot air heating experiment of the electro-magnesium alloy with cathodic protection.

magnesium oxide component with rugged mountain-like morphology appears on the magnesium alloy plate without cathodic protection, as shown in Figure 14. Granular powders are produced because of the impurities in magnesium alloys during smelting, as shown in Figure 15.

From Figure 16, some bulges of about 4 microns in diameter on the magnesium alloy plate with cathodic protection can be seen. EDS analysis shows that the magnesium alloy reacts with oxygen in the air, and the magnesium alloy produces local bulges. By comparing Figures 14 and 17 and using EDS analysis and comparison, it can be seen that there is no loose magnesium oxide component with rugged mountain-like morphology in the magnesium alloy plates in Figure 14, which verifies that cathodic protection can suppress the chemical reaction of magnesium alloy oxidation.

By EDS analysis (Figure 18), it can be concluded that by applying negative cathodic protection voltage, chemical reactions such as oxidation in magnesium alloy forming process can be effectively suppressed, which verifies the 


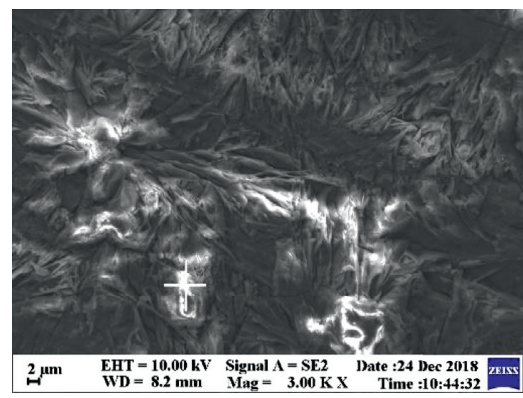

(a)

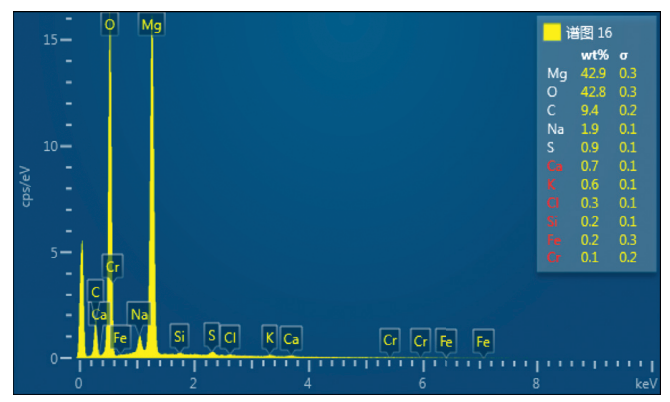

(b)

FIGURE 14: Superficial morphology experiment and EDS analysis of the directly heated magnesium alloy plate by SEM (3000 times) without cathodic protection voltage.

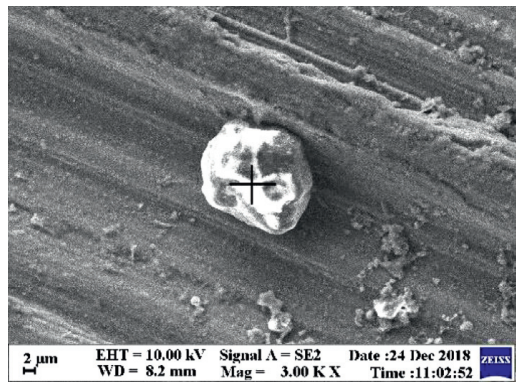

(a)

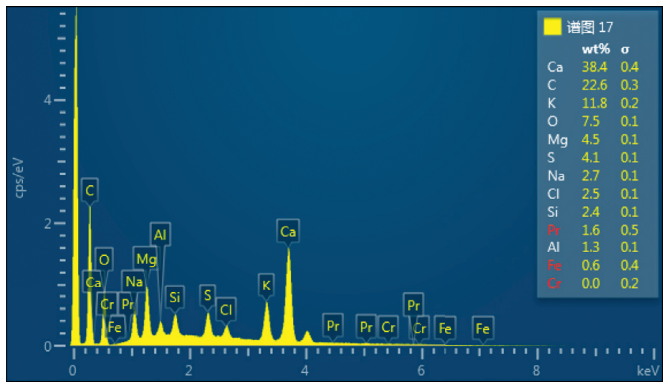

(b)

FIGURE 15: Superficial morphology experiment and EDS analysis of the directly heated magnesium alloy plate by SEM (3000 times) without cathodic protection voltage.

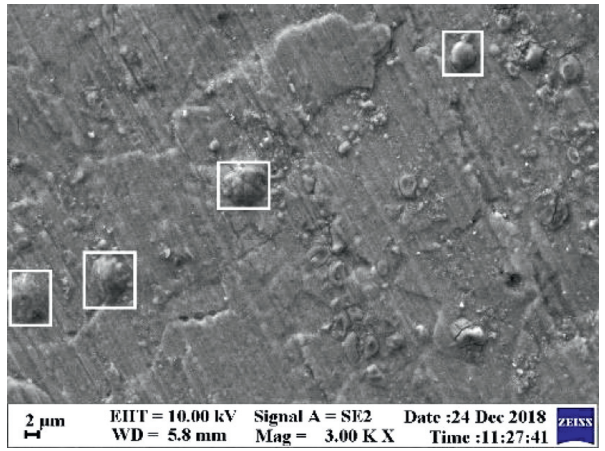

FIGURE 16: Experimental bulging phenomenon of SEM (3000 times) micromorphology of the magnesium alloy plate heated by cathodic protection.

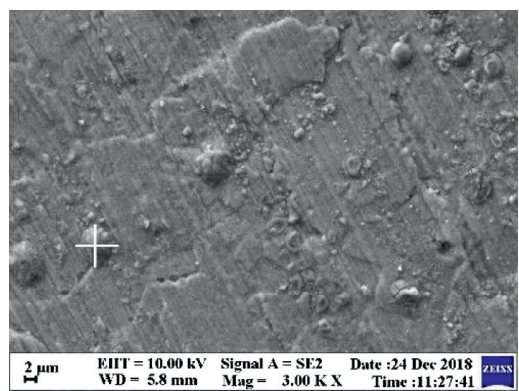

(a)

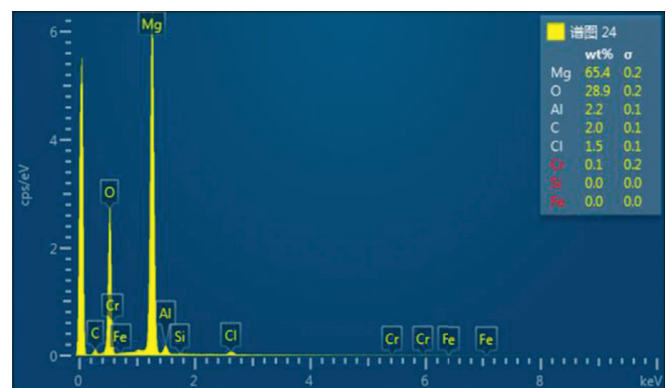

(b)

FIGURE 17: SEM (3000 times) micromorphology experiment and EDS analysis of the magnesium alloy plate heated by cathodic protection. 


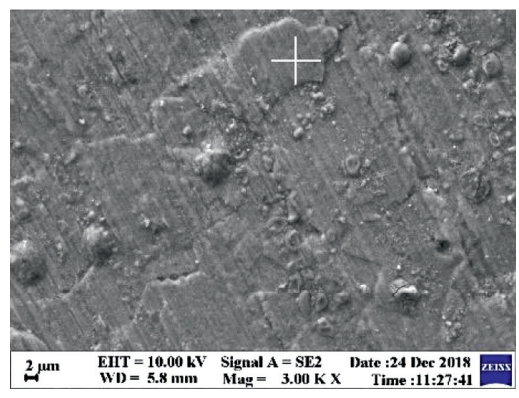

(a)

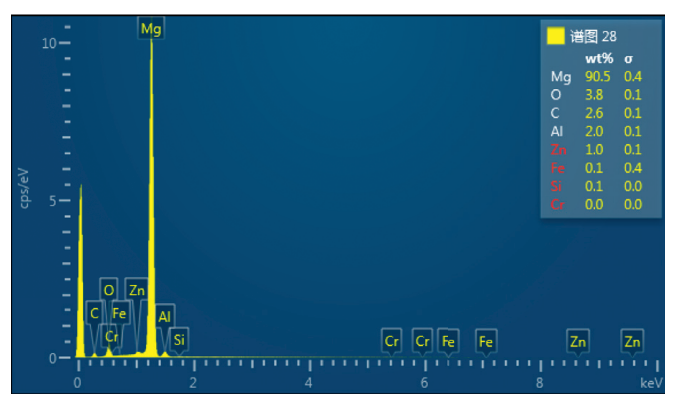

(b)

FIgURE 18: SEM (3000 times) micromorphology experiment and EDS analysis of the magnesium alloy plate after cathodic protection heating.

feasibility of the application of the electrochemical protection theory to active metal magnesium alloy forming.

To sum up, the thermal insulation of the magnesium alloy edges is achieved by circulating dry hot air, and the electrochemical reaction is achieved by applying cathode protection voltage to control the chemical reaction ability of the active magnesium alloy, which is helpful to improve the forming of magnesium alloy rolling.

\section{Conclusion}

(1) The temperature difference between the center and the edge of the magnesium alloy plate can be improved by the active temperature compensation method of the magnesium alloy edge during rolling, and then the edge crack can be reduced.

(2) The experimental results of cathodic protection voltage applied to the magnesium alloy verify that the chemical reaction of the magnesium alloy with other external substances can be effectively suppressed by the electrochemical method.

(3) During the production of the magnesium alloy, corrosion of the magnesium alloy resulting from the moisture in the air may directly affect the performance of the magnesium alloy. Therefore, the corresponding drying and filtering measures should be taken for the rolling equipment of the magnesium alloy.

\section{Data Availability}

All data generated or analyzed during this study are included within the article.

\section{Conflicts of Interest}

The authors declare that they have no conflicts of interest.

\section{Acknowledgments}

This work was financially supported by the Shanxi Provincial Key R\&D Projects (grant number 201803D121123), Shanxi Natural Science Foundation (grant number 201801D121086), and PHD Foundation (grant number 20182006).

\section{References}

[1] L.-Y. Chen, J.-Q. Xu, H. Choi et al., "Processing and properties of magnesium containing a dense uniform dispersion of nanoparticles," Nature, vol. 528, no. 7583, pp. 539-543, 2015.

[2] Z. Zeng, N. Stanford, C. H. J. Davies, J.-F. Nie, and N. Birbilis, "Magnesium extrusion alloys: a review of developments and prospects," International Materials Reviews, vol. 64, no. 1, pp. 27-62, 2019.

[3] A. Atrens, G.-L. Song, M. Liu et al., "Review of recent developments in the field of magnesium corrosion," Advanced Engineering Materials, vol. 17, no. 4, pp. 400-453, 2015.

[4] X. Rao, Y. Wu, X. Pei et al., "Influence of rolling temperature on microstructural evolution and mechanical behavior of AZ31 alloy with accumulative roll bonding," Materials Science and Engineering: A, vol. 754, pp. 112-120, 2019.

[5] H. Miyamoto, "Corrosion of ultrafine grained materials by severe plastic deformation," Materials Transactions, vol. 57, no. 5, pp. 559-572, 2016.

[6] Li Yang, L. Ma, Z. Jiang, Z. Huang, J. Lin, and Y. Ji, “Temperature field of temperature controlled roll for magnesium alloy," Rare Metal Materials and Engineering, vol. 48, no. 7, pp. 2074-2083, 2019.

[7] J. H. Lee, S. W. Lee, and S. H. Park, "Microstructural characteristics of magnesium alloy sheets subjected to high-speed rolling and their rolling temperature dependence," Journal of Materials Research and Technology, vol. 8, no. 3, pp. 3167$3174,2019$.

[8] K. Hagihara, Z. X. Li, M. Yamasaki, Y. Kawamura, and T. Nakano, "Strengthening mechanisms acting in extruded Mg-based long-period stacking ordered (LPSO)-phase alloys," Acta Materialia, vol. 163, no. 7, pp. 226-239, 2019.

[9] X. H. Shao, Z. Q. Yang, and X. L. Ma, "Strengthening and toughening mechanisms in $\mathrm{Mg}-\mathrm{Zn}-\mathrm{Y}$ alloy with a long period stacking ordered structure," Acta Materialia, vol. 58, no. 14, pp. 4760-4771, 2010.

[10] Y. Li, L. F. Ma, Z. Y. Jiang, Z. Huang, J. Lin, and Y. Ji, "Numerical simulation and experimental verification of temperature field in medium plate rolling of AZ31 magnesium alloy," Rare Metal Materials And Engineering, vol. 48, no. 7, pp. 2185-2192, 2019.

[11] B. Jiang, Q. Xiang, A. Atrens, J. F. Song, and F. S. Pan, "Influence of crystallographic texture and grain size on the corrosion behaviour of as-extruded Mg alloy AZ31 sheets," Corrosion Science, vol. 126, pp. 374-380, 2017.

[12] B. J. Wang, D. K. Xu, J. Sun, and E.-H. Han, "Effect of grain structure on the stress corrosion cracking (SCC) behavior of an as-extruded Mg-Zn-Zr alloy," Corrosion Science, vol. 157, pp. 347-356, 2019. 
[13] W. T. Jia, Y. Tang, Q. C. Le, and J. Z. Cui, “Air-cooling analysis of AZ31B magnesium alloy plate: experimental verification, numerical simulation and mathematical modeling," Journal of Alloys and Compounds, vol. 695, pp. 1838-1853, 2017.

[14] M. Esmaily, J. E. Svensson, S. Fajardo et al., "Fundamentals and advances in magnesium alloy corrosion," Progress in Materials Science, vol. 89, pp. 92-193, 2017.

[15] K. R. Hallam, P. C. Minshall, P. J. Heard, and P. E. J. Flewitt, "Corrosion of the alloys magnox AL80, magnox ZR55 and pure magnesium in air containing water vapour," Corrosion Science, vol. 112, pp. 347-363, 2016.

[16] M. Vlček, J. Čížek, F. Lukáč et al., "Hydrogen absorption in Mg-Gd alloy," International Journal of Hydrogen Energy, vol. 42, no. 35, pp. 22598-22604, 2017.

[17] M. Kamilyan, R. Silverstein, and D. Eliezer, "Hydrogen trapping and hydrogen embrittlement of Mg alloys," Journal of Materials Science, vol. 52, no. 18, pp. 11091-11100, 2017.

[18] A. Laureys, T. Depover, R. Petrov, and K. Verbeken, "Influence of sample geometry and microstructure on the hydrogen induced cracking characteristics under uniaxial load," Materials Science and Engineering: A, vol. 690, pp. 88-95, 2017.

[19] L. F. Zhou, Z. Y. Liu, W. Wu, X. G. Li, C. W. Du, and B. Jiang, "Stress corrosion cracking behavior of ZK60 magnesium alloy under different conditions," International Journal of Hydrogen Energy, vol. 42, no. 41, pp. 26162-26174, 2017.

[20] C. L. Cheng, Q. Lan, A. Wang, Q. Le, F. Yang, and X. Li, "Effect of $\mathrm{Ca}$ additions on ignition temperature and multistage oxidation behavior of AZ80," Metals, vol. 8, no. 10, p. 766, 2018.

[21] X. Zhang, Z. Y. Shen, N. Jian et al., “A novel complex oxide $\mathrm{TiVO}_{3.5}$ as a highly active catalytic precursor for improving the hydrogen storage properties of $\mathrm{MgH}_{2}$," International Journal of Hydrogen Energy, vol. 43, no. 52, pp. 23327-23335, 2018.

[22] K.-J. Jeon, H. R. Moon, A. M. Ruminski et al., “Air-stable magnesium nanocomposites provide rapid and high-capacity hydrogen storage without using heavy-metal catalysts," $\mathrm{Na}$ ture Materials, vol. 10, no. 4, pp. 286-290, 2011.

[23] E. Alasmar, I. Aubert, A. Durand et al., "Hydrogen generation from $\mathrm{Mg}-\mathrm{NdNiMg}_{15}$ composites by hydrolysis reaction," International Journal of Hydrogen Energy, vol. 44, no. 2, pp. 523-530, 2019.

[24] A. D. Gabbardo and G. S. Frankel, "Application of 2D pit growth method to Mg thin films: Part I. Initiation, growth and repassivation," Journal of the Electrochemical Society, vol. 166, no. 11, pp. C3254-C3265, 2019.

[25] O. A. Buryakovskaya, M. S. Vlaskin, and S. S. Ryzhkova, "Hydrogen production properties of magnesium and magnesium-based materials at low temperatures in reaction with aqueous solutions," Journal of Alloys and Compounds, vol. 785, pp. 136-145, 2019.

[26] S. Manivanna, S. S. Kumaran, A. Vallimanalan, R. Mahendran, and S. P. K. Babu, "Stress corrosion cracking of AZ91 + xCe alloy using proof ring test in ASTM D1384 and $\mathrm{NaCl}-\mathrm{K}_{2} \mathrm{CrO}_{4}$ solutions," Journal of Materials Engineering and Performance, vol. 28, no. 5, pp. 2552-2561, 2019.

[27] L. Oger, B. Malard, G. Odemer, L. Peguet, and C. Blanc, "Influence of dislocations on hydrogen diffusion and trapping in an Al-Zn-Mg aluminium alloy," Materials \& Design, vol. 180, Article ID 107901, 2019.

[28] H. Su, H. Toda, K. Shimizu, K. Uesugi, A. Takeuchi, and Y. Watanabe, "Assessment of hydrogen embrittlement via image-based techniques in $\mathrm{Al}-\mathrm{Zn}-\mathrm{Mg}-\mathrm{Cu}$ aluminum alloys," Acta Materialia, vol. 176, pp. 96-108, 2019.

[29] Q. M. Peng, Y. Sun, B. C. Ge et al., "In-situ atomic-scale phase transformation of $\mathrm{Mg}$ under hydrogen conditions," Journal of Physical Chemistry C, vol. 122, no. 34, pp. 19532-19539, 2018.

[30] S. Fajardo, J. Bosch, and G. S. Frankel, "Anomalous hydrogen evolution on AZ31, AZ61, and AZ91 magnesium alloys in unbuffered sodium chloride solution," Corrosion Science, vol. 146, pp. 163-171, 2019.

[31] E. Merson, P. Myagkikh, V. Poluyanov, D. Merson, and A. Vinogradov, "On the role of hydrogen in stress corrosion cracking of magnesium and its alloys: gas-analysis study," Materials Science and Engineering: A, vol. 748, pp. 337-346, 2019.

[32] A. Atrens, N. Winzer, and W. Dietzel, "Stress corrosion cracking of magnesium alloys," Advanced Engineering Materials, vol. 13, no. 1-2, pp. 11-18, 2011.

[33] M. Yang, X. B. Liu, Z. Y. Zhang, and Y. L. Song, "Stress corrosion behavior of AM50Gd magnesium alloy in Different environments rapidly at the bottom of pit, which was caused by corrosion," Metals, vol. 9, no. 5, p. 616, 2019.

[34] E. Schwarzenbock, T. Hack, M. Kolb, C. Engel, T. Heidenblut, and H. J. Maier, "Evolution of surface characteristics of two industrial $7 \mathrm{xxx}$ aluminium alloys exposed to humidity at moderate temperature," Surface and Interface Analysis, vol. 51, no. 12, pp. 1288-1297, 2019.

[35] H. G. Liu, F. Y. Cao, G.-L. Song et al., "Review of the atmospheric corrosion of magnesium alloys," Journal of Materials Science \& Technology, vol. 35, no. 9, pp. 2003-2016, 2019.

[36] M. Strebl and S. Virtanen, "Real-time monitoring of atmospheric magnesium alloy corrosion," Journal of the Electrochemical Society, vol. 166, no. 11, pp. C3001-C3009, 2018.

[37] F. Y. Cao, G.-L. Song, and A. Atrens, "Corrosion and passivation of magnesium alloys," Corrosion Science, vol. 111, pp. 835-845, 2016.

[38] D. Song, C. Li, N. N. Liang et al., "Simultaneously improving corrosion resistance and mechanical properties of a magnesium alloy via equal-channel angular pressing and post water annealing," Materials \& Design, vol. 166, Article ID 107621, 2019.

[39] S. Fintová, J. Drábiková, F. Pastorek et al., "Improvement of electrochemical corrosion characteristics of AZ61 magnesium alloy with unconventional fluoride conversion coatings," Surface \& Coatings Technology, vol. 357, pp. 638-650, 2019.

[40] D. Ottinger, M. Averyt, and D. Harris, "US consumption and supplies of sulphur hexafluoride reported under the greenhouse gas reporting program," Journal of Integrative Environmental Sciences, vol. 12, no. 1, pp. 5-16, 2015.

[41] S. Bartos, C. Laush, J. Scharfenberg, and R. Kantamaneni, "Reducing greenhouse gas emissions from magnesium die casting," Journal of Cleaner Production, vol. 15, no. 10, pp. 979-987, 2007.

[42] M. Deng, L. Q. Wang, D. Höche et al., "Clarifying the decisive factors for utilization efficiency of $\mathrm{Mg}$ anodes for primary aqueous batteries," Journal of Power Sources, vol. 441, Article ID 227201, 2019.

[43] Y. L. Wang, S. H. Zhang, P. Wang, S. Chen, Z. Lu, and W. Li, "Electropolymerization and corrosion protection performance of the $\mathrm{Nb}: \mathrm{TiO}_{2}$ nanofibers/polyaniline composite coating," Journal of the Taiwan Institute of Chemical Engineers, vol. 103, pp. 190-198, 2019. 
[44] M. Yang, X. B. Liu, Z. Y. Zhang, and Y. L. Song, "Stress corrosion behavior of AM50Gd magnesium alloy in different environments," Metals, vol. 9, no. 5, p. 616, 2019.

[45] J. A. Yuwono, C. D. Taylor, G. S. Frankel, N. Birbilis, and S. Fajardo, "Understanding the enhanced rates of hydrogen evolution on dissolving magnesium," Electrochemistry Communications, vol. 104, Article ID 106482, 2019.

[46] H. Miyamoto, M. Yuasa, M. Rifai, and H. Fujiwara, "Corrosion behavior of severely deformed pure and single-phase materials," Materials Transactions, vol. 60, no. 7, pp. 1243-1255, 2019.

[47] F. Y. Cao, C. Zhao, J. You, J. Hu, D. Zheng, and G.-L. Song, "The inhibitive effect of artificial seawater on magnesium corrosion," Advanced Engineering Materials, vol. 21, no. 8, Article ID 1900363, 2019.

[48] J. L. Ma, G. X. Wang, Y. Q. Li, C. H. Qin, and F. Z. Ren, "Electrochemical investigations on AZ series magnesium alloys as anode materials in a sodium chloride solution," Journal of Materials Engineering and Performance, vol. 28, no. 5, pp. 2873-2880, 2019.

[49] N. Shrestha, K. S. Raja, and V. Utgikar, "Mg-RE alloy anode materials for Mg-air battery application," Journal of the Electrochemical Society, vol. 166, no. 14, pp. A3139-A3153, 2019.

[50] H. Pan, K. Pang, F. Z. Cui et al., "Effect of alloyed Sr on the microstructure and corrosion behavior of biodegradable $\mathrm{Mg}$ Zn-Mn alloy in Hanks' solution," Corrosion Science, vol. 157, pp. 420-437, 2019.

[51] A. Soltan, M. S. Dargusch, Z. M. Shi, D. Gerrard, and A. Atrens, "Understanding the corrosion behaviour of the magnesium alloys EV31A, WE43B, and ZE41A," Materials and Corrosion, vol. 70, no. 9, pp. 1527-1552, 2019.

[52] O. A. Al-Rashed and A. A. Nazeer, "Ionic liquids with superior protection for mild steel in acidic media: effects of anion, cation, and alkyl chain length," Journal of Molecular Liquids, vol. 288, Article ID 111015, 2019.

[53] A. Bahmani, S. Arthanari, and K. S. Shin, "Corrosion behavior of Mg-Mn-Ca alloy: influences of $\mathrm{Al}, \mathrm{Sn}$ and $\mathrm{Zn}$," Journal of Magnesium and Alloys, vol. 7, no. 1, pp. 38-46, 2019.

[54] P. Gore, N. Birbilis, and V. S. Raja, "Temporal evolution of anodically activated cathodic kinetics on magnesium through atmospheric exposure," Corrosion, vol. 75, no. 6, pp. 687-692, 2019.

[55] J. Muldoon, C. B. Bucur, and T. Gregory, "Fervent hype behind magnesium batteries: an open call to synthetic chemists-electrolytes and cathodes needed," Angewandte Chemie International Edition, vol. 56, no. 40, pp. 1204612084, 2017.

[56] F. Y. Cao, Z. M. Shi, G.-L. Song, M. Liu, M. S. Dargusch, and A. Atrens, "Influence of hot rolling on the corrosion behavior of several Mg-X alloys," Corrosion Science, vol. 90, pp. 176191, 2015. 